\title{
Relationship between depression and inflammatory factors and brain-derived neurotrophic factor in patients with perimenopause syndrome
}

\author{
LI GUO $^{1}$, LIDONG REN ${ }^{2}$ and CAIXIA ZHANG ${ }^{1}$ \\ ${ }^{1}$ Department of Gynaecology and Obstetrics, Affiliated Hospital of Inner Mongolia Medical University; \\ ${ }^{2}$ Department of Breast, Thyroid, Hernia and Vascular Surgery, Inner Mongolia \\ People's Hospital, Hohhot, Inner Mongolia 010050, P.R. China
}

Received December 15, 2017; Accepted March 1, 2018

DOI: $10.3892 /$ etm.2018.5985

\begin{abstract}
The relationship of depression with levels of serum inflammatory factors and brain-derived neurotrophic factor (BDNF) in patients with perimenopausal syndrome was analyzed to investigate the predictive values of risk factors for depression in patients with perimenopausal syndrome. A total of 73 patients with perimenopausal syndrome were selected, and divided into the depression $(n=30)$ and non-depression $(n=43)$ groups. Results showed that the self-rating anxiety scale (SAS) and self-rating depression scale (SDS) scores in the depression group were significantly higher than those in the non-depression group $(\mathrm{P}<0.05)$. The serum BDNF level in the depression group was significantly lower than that in the non-depression group $(\mathrm{P}<0.05)$. The levels of serum inflammatory factors, including C-reactive protein (CRP), interleukin-6 (IL-6) and tumor necrosis factor- $\alpha$ (TNF- $\alpha$ ), in depression group were obviously higher than those in non-depression group $(\mathrm{P}<0.05)$. The correlation analyses of serum inflammatory factor and BDNF levels with depression showed that CRP and TNF- $\alpha$ were positively correlated with SDS score, while BDNF level was negatively correlated with SDS score. Logistic regression analyses revealed that menstrual status, chronic diseases, serum inflammatory factors (CRP, IL-6 and TNF- $\alpha$ ) and BDNF levels had independent predictive values for depression in patients with perimenopausal syndrome $(\mathrm{P}<0.05)$. In conclusion, levels of serum CRP, IL-6, TNF- $\alpha$ and BDNF can be used as judgment indicators for the severity of depression.
\end{abstract}

Correspondence to: Dr Li Guo, Department of Gynaecology and Obstetrics, Affiliated Hospital of Inner Mongolia Medical University, 1 Tongdaobei Road, Hohhot, Inner Mongolia 010050, P.R. China E-mail: azbop089@163.com

Key words: perimenopausal syndrome, depression, inflammatory factors, brain-derived neurotrophic factor

\section{Introduction}

With the increase of social pressure and the pace of life, mental illnesses, such as anxiety disorder, or even depression disorder, are increasingly common in perimenopause women due to hormonal metabolic disorders (1). Patients with perimenopausal syndrome present a series of symptoms, such as dysphoria, irascibility, mood swings and uncontrolled rage, which have a negative impact on the patients themselves and their families and lead to a lower quality of life $(2,3)$. The main reasons for these symptoms are the decline of ovarian function and significant reduction of endogenous estrogen secretion.

Previous research has shown that the level of brainderived neurotrophic factor (BDNF) in peripheral serum is closely related to the severity of depression (4), as it is an important component in neurotrophic factors. BDNF in the cells of brain tissue is composed of the tissues such as neurogliocyte and neuron in the brain tissue cells (5) and BDNF in peripheral serum is composed and secreted by blood platelets. When the composition or content of blood palates is stimulated by external or internal factors, a reduction or increase is observed in the synthesis and secretion of BDNF (6). When patients have depressive symptoms, a significant reduction in the content, synthesis and release of BDNF in peripheral serum is observed (7). As the inflammatory system is regulated and mediated by multiple inflammatory factors, antidepressants are often used in the treatment of the majority pf patients with depression disorder to lower the level of inflammatory factors and obtain a better treatment effect (8). However, more research data are necessary to support the related mechanisms.

Perimenopausal syndrome refers to the related clinical manifestations presented during the period from the first time of clinical manifestation of menopause to the time of the last menstruation. It is during this period that the probability of depression is increased (9). Therefore, the investigation on the risk factors associated with depression in perimenopausal syndrome patients can avoid or reduce the onset as much as possible or achieve the goal of early detection and early treatment. 
Table I. Comparisons of general data and main life events between depression group and non-depression group of patients with perimenopausal syndrome.

\begin{tabular}{lccc}
\hline Normal information & Depression group (n=30) & Non-depression group (n=43) & P-value \\
\hline Age (years) & $50.78 \pm 3.69$ & $49.01 \pm 2.41$ & 0.671 \\
Menstruation (normal/abnormal) & $4 / 26$ & $33 / 10$ & 0.003 \\
Work (work/no work) & $5 / 25$ & $38 / 5$ & 0.023 \\
Companion health (health/chronic diseases) & $18 / 12$ & $27 / 16$ & 0.058 \\
Marital status (good/divorced/widowed) & $5 / 23 / 2$ & $38 / 4 / 1$ & 0.001 \\
Self-chronic disease (yes/no) & $26 / 4$ & $16 / 27$ & 0.048 \\
\hline
\end{tabular}

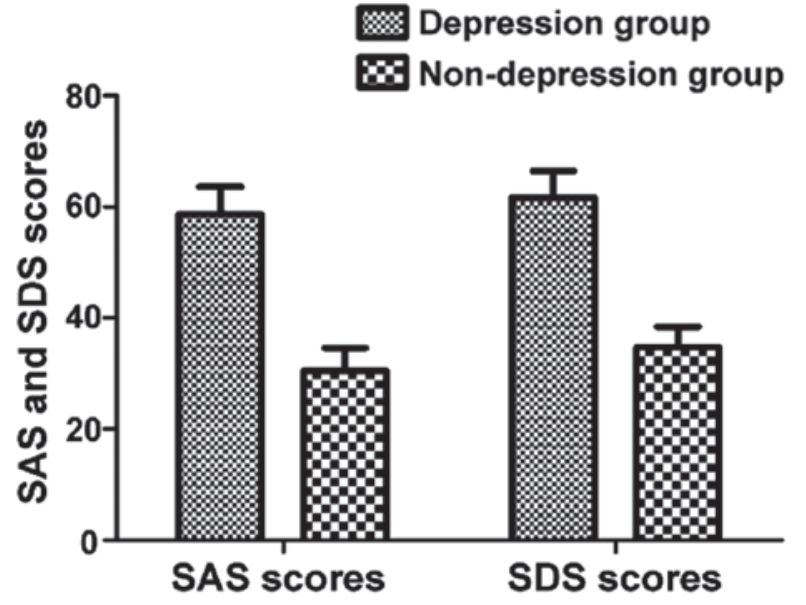

Figure 1. Comparisons of self-rating anxiety scale (SAS) scores and self-rating depression scale (SDS) scores between the depression and non-depression groups of patients with perimenopausal syndrome.

\section{Patients and methods}

General data. A total of 73 patients diagnosed with perimenopausal syndrome treated in the Affiliated Hospital of Inner Mongolia Medical University (Hohhot, China) from February, 2016 to January, 2017 were selected and divided into the depression $(n=30)$ and non-depression $(n=42)$ groups. The patients were aged 47-53 years, with an average age of $49.03 \pm 3.48$ years. All selected cases conformed to the related diagnostic requirements of perimenopausal syndrome specified in the 8th edition of Obstetrics and Gynaecology in China. The diagnosis of depression was based on the Hamiton's Depression Scale in 1959 (score >20 points) and the Chinese classification and diagnostic criteria of mental disorders. Exclusion criteria: Patients aged $<41$ years; patients who had taken related medicines that affected hormone level recently; patients with severe heart, liver or renal insufficiency; patients with malignant tumor; patients with mental disorders, such as anxiety, depression or schizophrenia in their past medical history; patients who did not sign the informed consent; patients without complete clinical data or who dropped out midway.

The study was approved by the Ethics Committee of Affiliated Hospital of Inner Mongolia Medical University. Written informed consent was provided by the patients.
Methods. Measurement of the level of inflammatory factors in peripheral serum [C-reactive protein (CRP), interleukin-6 (IL-6) and tumor necrosis factor- $\alpha$ (TNF- $\alpha$ )] and BDNF: Peripheral blood sample $(15 \mathrm{ml})$ was drawn from each of the included patients after fasting for solids and liquids for $10 \mathrm{~h}$ overnight, which was then centrifuged to obtain the upper serum. Serum $(10 \mathrm{ml})$ was used to measure the level of inflammatory factors in peripheral serum by immunoturbidimetry and the remaining $5 \mathrm{ml}$ was used to measure the level of BDNF by ELISA. All the reagents and instruments were provided by Hangzhou Biological Instruments Co., Ltd. (Hangzhou, China).

Calculation methods in self-rating anxiety scale (SAS) and self-rating depression scale (SDS): SDS scores include 20 items and the critical score is 50 points. The higher the total score is, the more obvious the depressive symptom will be. SAS score: $>50$ points, SAS $>60$ points (mild anxiety), $\leq 60$ points, SAS $<70$ points (moderate anxiety), $S A S \geq 70$ point (severe anxiety).

Statistical analysis. Statistical Product and Service Solutions (SPSS) 19.0 software (IBM, Armonk, NY, USA) was used for data processing, data collected were presented as mean $\pm \mathrm{SD}$, Chi-square test was used for the comparison of enumeration data, correlation analysis was conducted for the two factors, logistic analysis was conducted for the related risk factors, and $\mathrm{P}<0.05$ indicates that the difference was considered to be statistically significant.

\section{Results}

Comparison of general data and main life events between depression group and non-depression group of patients with perimenopausal syndrome. The results showed that the differences in the age and health condition of spouse between depression group and non-depression group had no statistical significance $(\mathrm{P}<0.05)$, while the number of cases having irregular menstruation, no work, being divorced or widowed and having chronic disease in the depression group was significantly higher than that in non-depression group, and the difference was statistically significant $(\mathrm{P}<0.05)$ (Table I).

Comparisons of SAS score and SDS score between the depression and non-depression groups of patients with perimenopausal syndrome. The results showed that SAS score 
Table II. Comparisons of inflammatory factor levels in peripheral serum between the depression and non-depression group of patients with perimenopausal syndrome.

\begin{tabular}{lcccr}
\hline Group & No. & CRP $(\mathrm{mg} / \mathrm{l})$ & TNF- $\alpha(\mathrm{pg} / \mathrm{ml})$ & $\mathrm{IL}-6(\mathrm{ng} / \mathrm{l})$ \\
\hline Depression group & 30 & $5.39 \pm 1.46$ & $13.67 \pm 3.19$ & $16.45 \pm 2.92$ \\
Non-depression group & 43 & $2.38 \pm 1.09$ & $7.06 \pm 3.56$ & $8.02 \pm 1.87$ \\
P-value & & 0.001 & 0.001 & 0.001 \\
\hline
\end{tabular}

CRP, C-reactive protein; TNF- $\alpha$, tumor necrosis factor- $\alpha$; IL-6, interleukin-6.

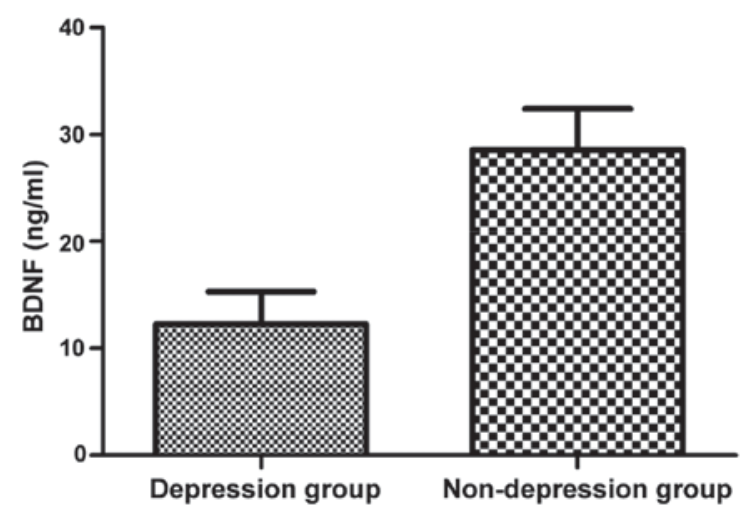

Figure 2. Comparison of brain-derived neurotrophic factor (BDNF) level in peripheral serum between depression group and non-depression group of patients with perimenopausal syndrome.

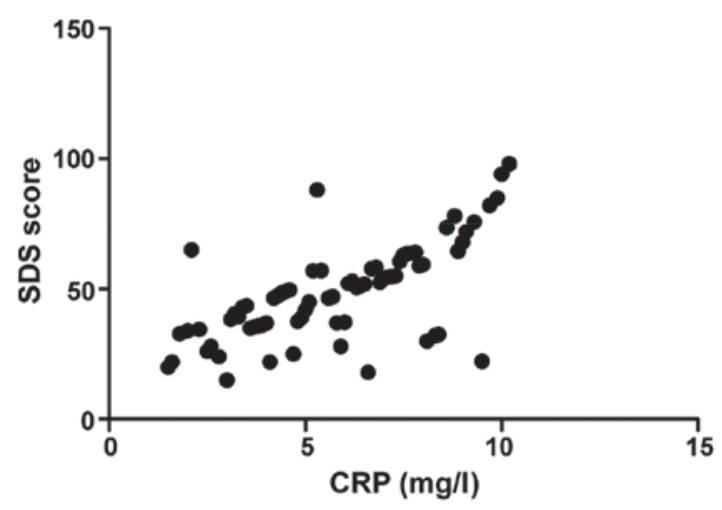

Figure 3. Correlation between C-reactive protein (CRP) and self-rating depression scale (SDS) score.

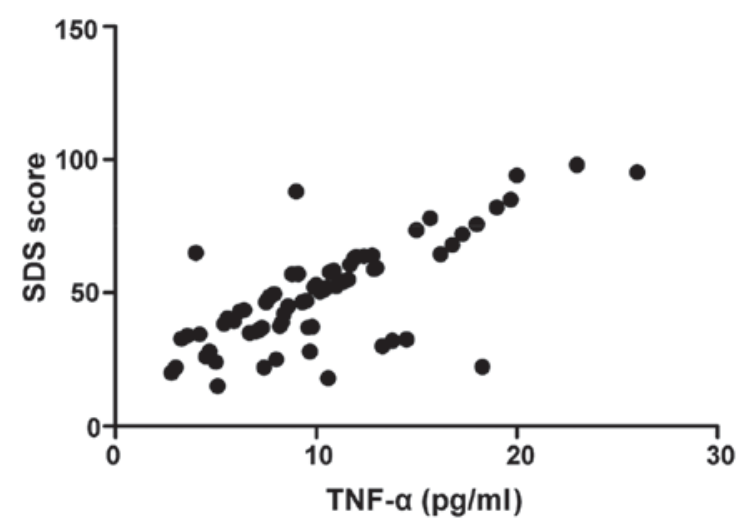

Figure 4. Correlation between tumor necrosis factor- $\alpha$ (TNF- $\alpha)$ and self-rating depression scale (SDS) score.
Table III. Logistic regression analysis of risk factors in predicting the onset of depression of patients with perimenopausal syndrome.

\begin{tabular}{lccc}
\hline Risk factors & P-value & OR value & $95 \%$ CI \\
\hline Age (years) & 0.109 & 1.095 & $0.986-1.157$ \\
Menstruation & 0.015 & 7.953 & $1.916-25.134$ \\
Work & 0.054 & 1.046 & $0.988-1.119$ \\
Companion health & 0.526 & 1.448 & $0.466-4.488$ \\
Marital status & 0.052 & 1.165 & $1.054-1.538$ \\
Self-chronic disease & 0.043 & 1.005 & $0.928-1.113$ \\
BDNF & 0.023 & 1.046 & $0.995-1.113$ \\
CRP & 0.001 & 1.004 & $0.995-1.321$ \\
IL-6 & 0.032 & 1.065 & $0.964-1.117$ \\
TNF- $\alpha$ & 0.029 & 1.054 & $0.984-1.065$ \\
\hline
\end{tabular}

BDNF, brain-derived neurotrophic factor; CRP, C-reactive protein; TNF- $\alpha$, tumor necrosis factor- $\alpha$; IL- 6 , interleukin- 6 .

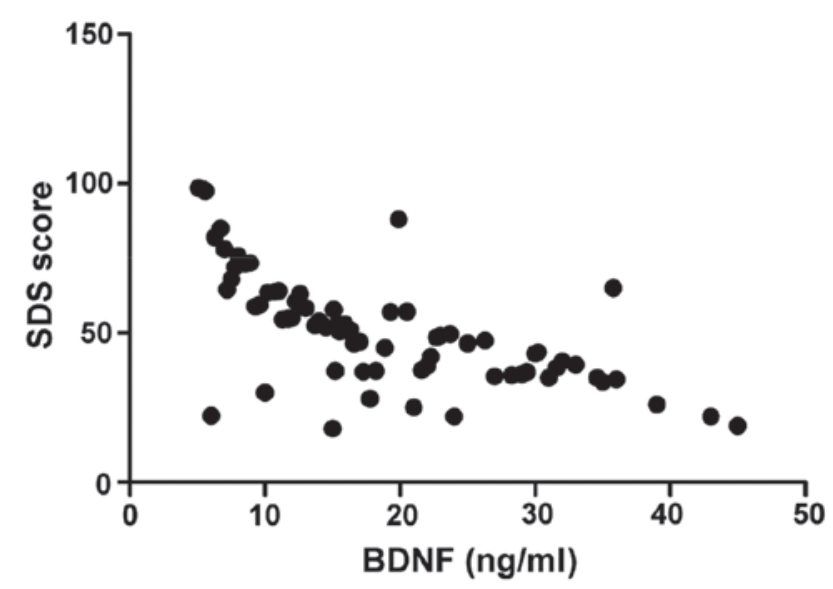

Figure 5. Correlation between brain-derived neurotrophic factor (BDNF) and self-rating depression scale (SDS) score.

and SDS score in depression group was significantly higher than those in non-depression group, and the differences were statistically significant $(\mathrm{P}<0.05)$ (Fig. 1).

Comparison of BDNF level in peripheral serum between the depression and non-depression groups of patients with perimenopausal syndrome. The results showed that BDNF 
level in peripheral serum in depression group was significantly lower than that in non-depression group, and the difference was statistically significant $(\mathrm{P}<0.05)$ (Fig. 2).

Comparison of inflammatory factor levels in peripheral serum between the depression and non-depression groups of patients with perimenopausal syndrome. The results showed that inflammatory factor levels (CRP, IL-6 and TNF- $\alpha$ ) in peripheral serum in depression group were significantly higher than those in non-depression group and the differences were statistically significant $(\mathrm{P}<0.05)$ (Table II).

Correlation analysis of inflammatory factor levels in peripheral serum and BDNF level with depression. The results showed CRP $(\mathrm{r}=0.793, \mathrm{P}<0.001)$ and $\mathrm{TNF}-\alpha(\mathrm{r}=0.682$, $\mathrm{P}<0.001)$ were all positively correlated with SDS score, while BDNF level was negatively correlated with SDS score $(r=-0.745, \mathrm{P}<0.001)$ (Figs. 3-5).

Logistic regression analysis of risk factors in predicting the onset of depression of patients with perimenopausal syndrome. The results indicated that the menstrual condition, chronic disease condition, inflammatory factor in peripheral serum (CRP, IL-6 and TNF- $\alpha$ ) and BDNP level had unique value in predicting the onset of depression of patients with perimenopausal syndrome $(\mathrm{P}<0.05)$ (Table III).

\section{Discussion}

As patients with depression are often in the psychological state of low mood, it is easy to cause substantial organ dysfunction, if not treated seriously for a long time. Depression is a common impaired mental function syndrome (10), especially in this rapid development world, the incidence of depression has become higher and higher with the increase of life pressure (11). Patients with perimenopausal syndrome, due to the metabolic disorders of hormone levels, are more likely to develop autonomic nerve dysfunction (12), which can be further developed into depression. Some patients have the symptom of depression from the beginning of perimenopause which may continue or repeat until menopause, while it may even continue for 5-10 years after menopause to overcome by patients (13), which will bring serious negative effects on patients themselves and their families as well as society. The most essential reason of having depression for patients with perimenopausal syndrome is the rapid reduction of endogenic estrogen content, resulting in moodiness, mainly manifested as irritability and depression (14). Thus, estrogen is often used in the clinical treatment of perimenopausal syndrome, which can effectively improve patients' depressed mood and promote their life quality (15). Relevant research data indicated that the use of exogenous estrogen in the treatment of perimenopausal syndrome can lower the inflammatory factor level and increase BDNF level, which can avoid onset of depression or effectively improve the severity of depression (16). However, this conclusion is necessary to be supported by more research data. BDNF is a protein component (17) with wide application existing in both central nervous system and peripheral nervous system of mammals, which can defend against anxiety and depression by mediating synapses by accelerating the differentiation of certain neurons (18). Hippocampus in the central nervous system is the main place where BDNF expression is produced. When BDNF content decreases, the patient will be found in an obvious depressive state (19). This study found that BDNF level in the group of perimenopausal syndrome patients with depression was significantly lower than that in the group without depression and BDNF was negatively correlated with SDS score, which was consistent with the conclusion.

The most common therapy for patients with depression is to use antidepressant. Research has shown that there is an obvious decline in patients' inflammatory factor level in peripheral serum during the use of antidepressant, thus inflammatory factor levels in peripheral serum are considered to be correlated with depression and its severity (20).

The conclusion from this study is that inflammatory factor levels in peripheral serum in depression group of patients with perimenopausal syndrome are significantly higher than those in non-depression group and inflammatory factor levels are positively correlated with SDS scores. In general, inflammatory factor levels in peripheral serum and BDNF level have important reference value in the depression occurred during perimenopause and can be regarded as the marker in evaluating the treatment effect of drug therapy on depression disorder. The decline of BDNF level in peripheral serum and increase of inflammatory factor levels demonstrate that the patient is more likely to develop depression disorder simultaneously or the depressive symptoms may be exacerbated. Therefore, BDNF level and inflammatory factor levels in peripheral serum have great significance in the diagnosis and treatment of perimenopausal syndrome.

\section{Acknowledgements}

Not applicable.

\section{Funding}

This work was supported in part by the Natural Science Foundation of Inner Mongolia (2017MS0828).

\section{Availability of data and materials}

All data generated or analyzed during this study are included in this published article.

\section{Authors' contributions}

LG was for study design, $\mathrm{LR}$ and $\mathrm{CZ}$ were for data collection and analysis, LG and CZ were for manuscript preparation. All authors read and approved the final manuscript.

\section{Ethics approval and consent to participate}

The study was approved by the Medical Ethics Committee of Affiliated Hospital of Inner Mongolia Medical University (no. INNER-MS-2015-IS-0043; Hohhot, China). Written informed consent was obtained from all the patients.

\section{Consent for publication}

Not applicable. 


\section{Competing interests}

The authors declare that they have no competing interests.

\section{References}

1. Zhou B, Sun X, Zhang M, Deng Y and Hu J: The symptomatology of climacteric syndrome: Whether associated with the physical factors or psychological disorder in perimenopausal/ postmenopausal patients with anxiety-depression disorder. Arch Gynecol Obstet 285: 1345-1352, 2012.

2. Gibbs Z, Lee S and Kulkarni J: Factors associated with depression during the perimenopausal transition. Womens Health Issues 23: e301-e307, 2013.

3. Graziottin A and Serafini A: Depression and the menopause: Why antidepressants are not enough? Menopause Int 15: 76-81, 2009.

4. Sun N, Yang CX, Liu ZF, Li XR, Xu Y and Zhang KR: Effects of polymorphisms of serotonin transporter promoter (5-HTTLPR) and brain derived neurotrophic factor gene (G196A rs6265) on the risk of major depressive disorder in the Chinese Han population. Eur Rev Med Pharmacol Sci 20: 1852-1859, 2016.

5. Studd JW: A guide to the treatment of depression in women by estrogens. Climacteric 14: 637-642, 2011.

6. Harte-Hargrove LC, Maclusky NJ and Scharfman HE: Brain-derived neurotrophic factor-estrogen interactions in the hippocampal mossy fiber pathway: Implications for normal brain function and disease. Neuroscience 239: 46-66, 2013.

7. Scharfman HE and MacLusky NJ: Estrogen and brain-derived neurotrophic factor (BDNF) in hippocampus: Complexity of steroid hormone-growth factor interactions in the adult CNS. Front Neuroendocrinol 27: 415-435, 2006.

8. Aguirre CC and Baudry M: Progesterone reverses 17betaestradiol-mediated neuroprotection and BDNF induction in cultured hippocampal slices. Eur J Neurosci 29: 447-454, 2009.

9. Strauss JR: The reciprocal relationship between menopausal symptoms and depressive symptoms: A 9-year longitudinal study of American women in midlife. Maturitas 70: 302-306, 2011.

10. Tuomisto H, Salo P, Saarinen R, Kalleinen N and Polo-Kantola P: The association of serum oestradiol level, age, and education with cognitive performance in peri- and late postmenopausal women. Maturitas 71: 173-179, 2012.

11. Song IU, Kim YD, Kim JS, Lee KS and Chung SW: Can high-sensitivity $\mathrm{C}$-reactive protein and plasma homocysteine levels independently predict the prognosis of patients with functional disability after first-ever ischemic stroke? Eur Neurol 64 304-310, 2010
12. Kroenke K, Spitzer RL and Williams JB: The PHQ-9: Validity of a brief depression severity measure. J Gen Intern Med 16 : 606-613, 2001.

13. Wojnar M, Drózdz W, Araszkiewicz A, Szymański W, Nawacka-Pawlaczyk D and Urbański R: Prevalence of depressive disorders among perimenopausal women seeking gynecological services. Psychiatr Pol 37: 811-824, 2003 (In Polish).

14. Huerta R, Mena A, Malacara JM and de León JD: Symptoms at the menopausal and premenopausal years: Their relationship with insulin, glucose, cortisol, FSH, prolactin, obesity and attitudes towards sexuality. Psychoneuroendocrinology 20: 851-864, 1995.

15. Komesaroff PA, Esler MD and Sudhir K: Estrogen supplementation attenuates glucocorticoid and catecholamine responses to mental stress in perimenopausal women. J Clin Endocrinol Metab 84: 606-610, 1999.

16. Miklowitz DJ: Delinquency, depression, and psychosis among adolescents in foster care: What holds three heads together? J Am Acad Child Adolesc Psychiatry 53: 1251-1253, 2014

17. Reinhardt JP, Horowitz A, Cimarolli VR, Eimicke JP and Teresi JA: Addressing depression in a long-term care setting: A phase II pilot of problem-solving treatment. Clin Ther 36: 1531-1537, 2014.

18. Kwasky AN and Groh CJ: Vitamin D, depression and coping selfefficacy in young women: Longitudinal study. Arch Psychiatr Nurs 28: 362-367, 2014.

19. Rosario JL, Bezerra Diógenes MS, Mattei R and Leite JR: Differences and similarities in postural alterations caused by sadness and depression. J Bodyw Mov Ther 18: 540-544, 2014.

20. Hayden EP, Olino TM, Mackrell SV, Jordan PL, Desjardins J and Katsiroumbas P: Cognitive vulnerability to depression during middle childhood: Stability and associations with maternal affective styles and parental depression. Pers Individ Dif 55: 892-897, 2013 